\title{
Predictive Value of Psychological Scales for Hospitalization of Elderly Suicide Attempters
}

\author{
Eundo Kim ${ }^{1}$ Ki Young Jeong, ${ }^{2,3}$ Jong Seok Lee, ${ }^{2,3}$, Han Sung Choi ${ }^{2,3}$ \\ ${ }^{1}$ Department of Emergency Medicine, Kyung Hee University Hospital at Gangdong, Seoul, ${ }^{2}$ Department of Emergency Medicine, Kyung \\ Hee University Medical Center, Seoul, ${ }^{3}$ Department of Emergency Medicine, Kyung Hee University School of Medicine, Seoul, Korea
}

Corresponding Author:

Ki Young Jeong, MD, PhD

Department of Emergency Medicine,

Kyung Hee University Medical

Center, 23 Kyungheedae-ro,

Dongdaemun-gu, Seoul 02447,

Korea

Tel: +82-2-958-8990

Fax: +82-2-958-9689

E-mail: fly10bird@gmail.com

Received: September 25, 2017

Revised: October 23, 2017

Accepted: October 25, 2017
Background: Suicide lethality and intent are known potential determinants of hospitalization, and high degrees of these factors are discriminating characteristics of elderly suicide attempters. We evaluated their ability to predict hospitalization in elderly suicide attempters. Methods: Via medical charts and interview questionnaires, data were collected retrospectively on suicide attempters aged 65 years and older who presented to the Emergency Department (ED) from January 2012 to December 2016. Subjects were divided into hospitalization and discharge groups. Degrees of suicide lethality and intent were evaluated using the Risk-Rescue Rating Scale (RRRS) and the Suicide Intent Scale (SIS), respectively. The predictive abilities of these scales for hospitalization were assessed using logistic regression analysis and compared with the area under the receiver operating characteristic curve. Results: Of 168 patients, 110 (65\%) were hospitalized. The RRRS and SIS scores for hospitalized patients were significantly higher; both scales were identified as independent predictors of hospitalization. The predictive performance of the RRRS for hospitalization was significantly superior to that of the SIS. Among SIS subscales, only the SIS part 2 was found to be an independent predictor of hospitalization. Its performance tended to be higher than the SIS, and comparable to the RRRS. The RRRS and SIS part 2 had greater sensitivity for predicting hospitalization than specificity. Conclusion: For elderly suicide attempters in the ED, the RRRS is a useful predictor of hospitalization. Further investigations based on the feasibility of the SIS part 2 as a predictor are needed.

Key Words: Elderly, Suicide, Scales, Hospitalization, Emergency Department

\section{INTRODUCTION}

Suicide is one of the leading causes of death, affecting more than 12 of every 100,000 people worldwide each year". Suicide affects people of all age groups, but has become a particularly important health issue in many countries for elderly people over 65 years of age. Indeed, high mortality rates following suicide attempts (SAs), as well as high suicide rates, have been reported in the elderly population ${ }^{2)}$. However, because the risk for SAs in elderly people is known to be associated with complex interactions of diverse factors, such as physical and mental illness, isolation, bereavement, and degraded socioeconomic support, a thorough investigation of the characteristics of SAs in elderly people is challenging for clinicians ${ }^{3-5)}$. The accelerating average age of the population, coupled with an increasing average lifespan, contributes to steady maintenance of high suicide rates in the elderly population ${ }^{6}$. Therefore, SAs in elderly people will remain an important public health issue that clinicians must address.

During management of elderly suicide attempters in emergency settings, the decision to hospitalize is as crucial a dimension for ascertaining the patients' prognosis and outcome as acute stabilization of their concurrent injuries ${ }^{7}$. Recent studies have reported that hospitalization itself correlates with reduction of interpersonal conflict, immediate suicide risk, and SA incidence after hospital discharge, demonstrating that hospitalization might be an important psychiatric intervention for suicide attempters ${ }^{8-10)}$. Additionally, elderly suicide attempters are at risk of aggravation of prior medical and physical conditions caused by injuries following a $S A$; thus, with respect to these patients, it might be more important to consider hospitalization a means of therapeutic intervention. However, thus far, validated guidelines for hospitalization in elderly suicide attempters have not been available. Therefore, the decision to hospitalize made in the Emergency Department (ED) tends to depend on the treating clinician's judgment of the SA's severity. 
Suicide lethality and intent are closely associated with SA severity as evaluated by clinicians ${ }^{11)}$. The degrees of these two factors appear to vary according to the age of the suicide attempters. Typically, high suicidal lethality and intent are associated with increasing age and are thus discriminating characteristics of elderly suicide attempters when compared to other age groups ${ }^{5,12,13)}$. In addition, the degree of suicide lethality and intent can be clinically evaluated in the ED using psychological scales; the Risk-Rescue Rating Scale (RRRS) and the Suicide Intent Scale (SIS) are commonly used to assess suicide lethality and intent, respectively ${ }^{14,15)}$. Therefore, we hypothesized that these age-related factors might be more valuable as predictors of hospitalization in elderly suicide attempters. Although suicide lethality and intent have long been acknowledged as potential determinants of hospitalization, studies are lacking on the degree to which adherence to these factors affects a clinician's decision to hospitalize elderly suicide attempters ${ }^{11,16,17)}$.

In the present study, we investigated the predictive ability of the lethality and intent dimensions for hospitalization of elderly suicide attempters in emergency settings. Using the measured scores of psychological scales reflecting these 2 dimensions (the RRRS and SIS), we evaluated which scale showed better predictive performance for hospitalization. Furthermore, in previous studies, the SIS subscales have been investigated to assess the risk of suicide; however, these subscales showed a discrepancy in suicide prediction ${ }^{18-22)}$. Therefore, we hypothesized that these subscales might bear no resemblance to one another in their predictive ability for hospitalization, and we additionally evaluated their predictive performance for hospitalization.

\section{MATERIALS AND METHODS}

\section{Study Design and Population}

This study was conducted on suicide attempters aged 65 years or older who presented to the ED of an urban academic tertiary-care hospital from January 1, 2012, to December 31, 2016. A suicide attempter is defined as a person who inflicted self-harm with the clear intention to end his/her life ${ }^{23)}$. Data were collected retrospectively from medical records and from interview questionnaires conducted by psychiatric researchers. Excluded from the study were all suicide attempters who were dead on arrival or died during ED management, and those who did not regain consciousness. The study protocol and the exemption of informed consent were approved by the Institutional Review Board of Kyung Hee University (approval number: 2017-090-001).

\section{Study Protocol}

Primary management of patients seen in the ED after a
SA was conducted by ED residents under the supervision of board-certified emergency physicians. They began treating the physical injuries caused by the suicidal behavior and collected clinical information related to the SA. As the suicide attempter's condition stabilized, the patient was referred to on-duty psychiatric residents. Psychiatric residents thoroughly evaluated the seriousness of the patient's symptoms in relation to his/her mental health problem and devised an appropriate plan for psychiatric treatment. Whenever possible, all RRRS and SIS ratings were completed by trained residents at the time of assessment during ED management. All patients were interviewed by the trained psychiatric researcher while in the ED. The interview using a standardized questionnaire form was designed to evaluate the comprehensive circumstances of the suicide attempter, including risk factors for SAs, socioeconomic status, and medical or psychiatric conditions. All clinical information was collected from the patient himself/herself and from at least one of his/her guardians.

After ED management of suicide attempters, clinical decisions may result in hospitalization in the medical/surgical or psychiatric ward, or in discharge from the ED. This decision is made based on the clinical judgment of the emergency physicians, considering the need for medical/physical treatment, risk of impending or subsequent SAs, and need for referral to a psychiatric specialist for follow-up. When a patient's injury induced by an SA was prioritized in his/her management, he/she was assigned to the medical/surgical ward by emergency physicians, and the patient's management was then conducted in parallel with psychiatric consultation during hospitalization. Alternately, if the emergency physicians judged that the patient did not require medical/surgical intervention, they cooperated with psychiatrists to determine the necessity of admission to the psychiatric ward.

\section{Measurement and Assessments}

We collected data including patients' sociodemographic, medical, and prior psychiatric characteristics, as well as the circumstances and clinical features of the suicidal behavior. Circumstances and clinical features included alcohol use during the SA, SA method, degree of suicidal lethality and intent, and final psychiatric diagnosis.

Suicide lethality, defined as the probability of inflicting a medical or physical injury followed by SA, was evaluated using the RRRS; the psychometric properties of this scale are well established ${ }^{4,24)}$. The scale has 2 subscales, "risk factors" and "rescue factors," each of which comprise 5 items (with a point value of 1, 2, or 3). Risk factors include the method used, level of consciousness, toxicity, reversibility, and treatment required, whereas rescue factors include location, person initiating rescue, probability of discovery, accessibility for rescue, and delay until discovery. The total number of points for each subscale is converted to a scale ranging in 
score from 1 to 5 . The final RRRS score is expressed as (risk score)/(sum of risk and rescue scores), with higher scores indicating more severe lethality.

Suicide intent, referring to the seriousness or intensity of a patient's subjective desire to die at the time of the SA, was evaluated using the $\operatorname{SIS}^{15)}$. This scale is divided into 2 sections. The SIS part 1 reflects the objective circumstances related to the SA (8 items), and the SIS part 2 is a self-report of the suicide attempter's own feelings and thoughts (7 items). The former includes isolation, timing, precautions against discovery, actions to seek help during or after the act, actions taken in anticipation of death, degree of preparation, presence of a suicide note, and communication of intent before the act. The latter includes the alleged purpose of the SA, expectations of fatality, conception of the method's lethality, seriousness of desire to end life, attitude toward living and dying, conception of rescuability, and degree of premeditation. Each item is rated on a 3-point scale $(0,1$, or 2$)$. The reliability and validity of the scale are well validated, with higher SIS scores representing higher suicide intent ${ }^{15)}$. If the suicide attempter was not stable enough to respond to interviews with physicians due to his/her physically/mentally stressful condition after SA, only the SIS part 1 (objective circumstances) score was calculated, and this was substituted for the total SIS score. Furthermore, because these two subscales showed a discrepancy in their prediction of suicide and in their correlation with groups of psychiatric symptoms and exhibited the opposite dimension of impulsivity on factors analyses using their items ${ }^{20-22)}$, the score of each SIS subscale was measured for analysis as well as the total SIS score.

\section{Data Analysis}

The elderly suicide attempters included in the study were categorized into 2 groups, hospitalization and discharge, according to whether they were hospitalized (medical/surgical and psychiatric wards) or discharged from the ED.

Continuous variables, expressed as means \pm standard de-

Table 1. Sociodemographic characteristics of enrolled patients

\begin{tabular}{|c|c|c|c|}
\hline Variable & Discharge group $(\mathrm{n}=58)$ & Hospitalization group $(\mathrm{n}=110)$ & p-value \\
\hline Age (yr) & $69.4 \pm 4.2$ & $70.5 \pm 3.6$ & 0.488 \\
\hline Sex & & & 0.375 \\
\hline Male & $38(65.5)$ & $61(55.5)$ & \\
\hline Female & $19(34.5)$ & $49(44.5)$ & \\
\hline Marital status & & & 0.014 \\
\hline Single & $2(3.4)$ & $0(0)$ & \\
\hline Married & $50(86.2)$ & $66(60.0)$ & \\
\hline Separated & $2(3.4)$ & $14(12.7)$ & \\
\hline Widowed & $4(6.9)$ & $30(27.3)$ & \\
\hline Educational status & & & 0.781 \\
\hline Elementary school and lower & $31(53.4)$ & $61(55.5)$ & \\
\hline Middle school & $18(31.0)$ & $24(21.8)$ & \\
\hline High school & $9(15.5)$ & $22(20.0)$ & \\
\hline University and higher & $0(0)$ & $3(2.7)$ & \\
\hline Cohabitant & & & 0.982 \\
\hline Living with a helper & $49(84.5)$ & $92(83.6)$ & \\
\hline Living alone & $9(15.5)$ & $18(16.4)$ & \\
\hline Employment & & & 0.824 \\
\hline Yes & $25 / 57(43.9)$ & $45 / 108(41.7)$ & \\
\hline No & $32 / 57(56.1)$ & $63 / 108(58.3)$ & \\
\hline Insurance & & & 0.705 \\
\hline National health insurance & $53(91.4)$ & $105(95.5)$ & \\
\hline Medical care & $5(8.6)$ & $4(3.6)$ & \\
\hline None & $0(0)$ & $1(0.9)$ & \\
\hline Chronic disease & & & 0.791 \\
\hline Yes & $47(81.0)$ & $86(78.2)$ & \\
\hline No & $11(19.0)$ & $24(21.8)$ & \\
\hline
\end{tabular}

Values are presented as mean \pm standard deviation or number (\%). 
viations, were compared between the groups using a Student t-test; categorical variables are expressed as absolute values and percentages and were compared using the chi-square or Fisher exact test. To evaluate the independence of the psychological scales as predictors of hospitalization, multivariate logistic regression analysis was performed with variables showing a trend toward significance in univariate analysis $(p<0.2)$.

Subsequently, receiver operating characteristic (ROC) curve analyses were used to determine the psychological scales' predictive performance for hospitalization, and performance was evaluated using areas under the curve (AUCs). The AUCs of the psychological scales were additionally compared to each other to determine which scale had superior performance. The optimal cutoff values yielding the maximal sum of the psychological scales' sensitivity and specificity for hospitalization were identified using the ROC curves. The sensitivity and specificity were measured at each cutoff value.

All statistically significant differences were defined as $\mathrm{p}<0.05$. IBM SPSS Statistics ver. 21.0 (IBM Co., Armonk, NY, USA) and STATA SE ver. 10.1 (Stata Corp., College Station, TX, USA) were used for data analysis.

\section{RESULTS}

A total of 168 elderly subjects presenting in the ED with SAs were included in the study. Among these, $110(65 \%)$ were included in the hospitalization group.

There were no significant differences in sociodemographic characteristics between the hospitalization and discharge groups except for marital status (Table 1). The overall psychiatric characteristics of the included patients are shown in Table 2; of the total subjects, $64.6 \%$ had a depressive disorder, $69.5 \%$ were being treated for their first SA, and $48.2 \%$ had committed their SA under the influence of alcohol. No significant differences in the SA-related psychiatric characteristics of included patients were observed in the groups.

The differences between the 2 groups in the SA methods used were not significant (Table 3). SA methods known to be lethal were used in $31.8 \%$ of patients in the hospitalization group and $27.5 \%$ of those in the discharge group $(p=0.715)$. Drug overdose and pesticide/caustic poisoning were the 2 highest-ranked SA methods in both groups; although drug overdose was relatively more prevalent in the discharge group, pesticide/caustic poisoning was relatively more prevalent in the hospitalization group. Furthermore, there were no significant differences observed in the final psychiatric diagnoses between the groups; the psychiatric disorders ranked first and second in both groups corresponded to each other, namely, depression and adjustment disorder, respectively (Table 3).

The RRRS and SIS were administered to 166 and 158 patients, respectively. Their measured values are shown in Table 3. The scores of both psychological scales were significantly higher in the hospitalization group ( $p<0.001$ for the RRRS and $p=0.006$ for the SIS). Furthermore, the SIS part 1 and part 2 were measured in 158 and 129 patients, respectively;

Table 2. Psychiatric characteristics related to suicide attempts in enrolled patients

\begin{tabular}{|c|c|c|c|}
\hline Variable & Discharge group $(\mathrm{n}=58)$ & Hospitalization group $(n=110)$ & p-value \\
\hline Previous psychiatric treatment & & & 0.823 \\
\hline Yes & $28(48.3)$ & $49(44.5)$ & \\
\hline No & $30(51.7)$ & $61(55.5)$ & \\
\hline Previous psychiatric hospitalization & & & 0.519 \\
\hline Yes & $12 / 56(21.4)$ & $30 / 107(28.0)$ & \\
\hline No & $44 / 56(78.6)$ & $77 / 107(72.0)$ & \\
\hline Previous depression diagnosis & & & 0.586 \\
\hline Yes & $39 / 57(68.4)$ & $69(62.7)$ & \\
\hline No & $18 / 57(31.6)$ & $41(37.3)$ & \\
\hline Psychiatric family history & & & 0.777 \\
\hline Yes & $8 / 55(14.5)$ & 19/106 (17.9) & \\
\hline No & $47 / 55(85.5)$ & $87 / 106(82.1)$ & \\
\hline Previous SA & & & 0.918 \\
\hline Yes & $17(29.3)$ & $34 / 109(31.2)$ & \\
\hline No & $41(70.7)$ & $75 / 109(68.8)$ & \\
\hline Alcohol use during SA & & & 0.939 \\
\hline Yes & $27(46.6)$ & $54(49.1)$ & \\
\hline No & $31(53.4)$ & $56(50.9)$ & \\
\hline
\end{tabular}

Values are presented as number (\%).

$\mathrm{SA}$, suicide attempt. 
Table 3. Clinical characteristics related to suicide attempts in enrolled patients

\begin{tabular}{|c|c|c|c|}
\hline Variable & Discharge group $(n=58)$ & Hospitalization group $(n=110)$ & p-value \\
\hline Method of suicide attempt & & & 0.211 \\
\hline Drug overdose & $39(67.2)$ & $59(53.6)$ & \\
\hline Pesticide or caustic poisoning & $8(13.8)$ & $24(21.8)$ & \\
\hline Gas inhalation & $0(0.0)$ & $2(1.8)$ & \\
\hline Hanging or strangulation & $4(6.9)$ & $8(7.3)$ & \\
\hline Cutting or piercing & $3(5.2)$ & $14(12.8)$ & \\
\hline Jumping or other & $4(6.9)$ & $3(2.7)$ & \\
\hline Final diagnosis & & & 0.361 \\
\hline Anxiety disorder & $1(1.7)$ & $3(2.7)$ & \\
\hline Depressive disorder & $41(70.7)$ & $71(64.6)$ & \\
\hline Bipolar disorder & $1(1.7)$ & $2(1.8)$ & \\
\hline Schizophrenia & $0(0)$ & $2(1.8)$ & \\
\hline Adjustment disorder & $12(20.7)$ & $29(26.4)$ & \\
\hline Personality disorder & $2(3.5)$ & $0(0)$ & \\
\hline Cognitive disorder & $1(1.7)$ & $2(1.8)$ & \\
\hline Others & $0(0)$ & $1(0.9)$ & \\
\hline RRRS* & $30.68 \pm 14.43$ & $43.17 \pm 12.06$ & $<0.001$ \\
\hline Risk score & $1.54 \pm 0.71$ & $2.76 \pm 1.04$ & $<0.001$ \\
\hline Rescue score & $3.42 \pm 1.14$ & $3.31 \pm 0.92$ & 0.502 \\
\hline $\operatorname{SIS}^{\dagger}$ & $9.48 \pm 5.77$ & $13.12 \pm 5.99$ & 0.006 \\
\hline Part 1 (objective circumstance) ${ }^{\dagger}$ & $5.78 \pm 3.83$ & $7.22 \pm 4.07$ & 0.069 \\
\hline Part 2 (self-report) ${ }^{\ddagger}$ & $4.09 \pm 3.62$ & $6.90 \pm 3.96$ & 0.004 \\
\hline
\end{tabular}

Values are presented as number (\%) or mean \pm standard deviation.

RRRS, Risk-Rescue Rating Scale; SIS, Suicide Intent Scale.

*The RRRS was administered to 166 patients (56 patients in the discharge group and 110 patients in the hospitalization group). ${ }^{\dagger}$ Both the SIS and SIS part 1 were administered to 158 patients (52 patients in the discharge group and 106 patients in the hospitalization group).

${ }^{\ddagger}$ The SIS part 2 was administered to 129 patients (42 patients in the discharge group and 87 patients in the hospitalization group).

the score of the SIS part 1 tended to be higher $(p=0.069)$, whereas that of the SIS part 2 was significantly higher $(p=$ $0.004)$ in the hospitalization group than in the discharge group (Table 3). Using variables with $p<0.2$ in univariate analyses, multivariate logistic regression analyses between the psychological scales (separate analyses between lethality [RRRS] and intent [SIS and SIS subscales]) were performed. The RRRS, SIS, and SIS part 2 were identified as independent predictors of hospitalization in elderly suicide attempters (Table 4). The intraclass correlation coefficient between independent scales, which were log transformed, was 0.786 (95\% confidence interval[Cl], 0.667-0.877; $p<0.001$ ).

The predictive performance of the RRRS, SIS, and SIS part 2 for hospitalization was $0.788(95 \% \mathrm{Cl}, 0.697-0.878), 0.664$ $(95 \% \mathrm{Cl}, 0.562-0.765)$, and $0.715(95 \% \mathrm{Cl}, 0.608-0.821)$, respectively. Figure 1 shows the discriminative performance of the psychological scales for predicting hospitalization. The AUC of the RRRS was significantly higher than that of the SIS ( 0.777 vs. $0.662, p=0.042$ ) (Fig. $1 \mathrm{~A})$. In addition, the AUC of the SIS part 2 was comparable to that of the RRRS $(0.703$ vs. $0.765, p=0.315$ ) (Fig. 1B), whereas it tended to be higher
Table 4. Psychological scales as predictors of hospitalization in the multivariate logistic regression analyses

\begin{tabular}{lccr}
\hline \hline Variable & OR & $95 \%$ CI & p-value \\
\hline RRRS & 1.095 & $1.043-1.149$ & $<0.001$ \\
SIS & 1.115 & $1.020-1.219$ & 0.017 \\
RRRS & 1.087 & $1.044-1.131$ & $<0.001$ \\
SIS part 1 & 1.071 & $0.945-1.214$ & 0.282 \\
RRRS & 1.072 & $1.025-1.119$ & 0.001 \\
SIS part 2 & 1.237 & $1.058-1.447$ & 0.008 \\
\hline
\end{tabular}

Adjusted for variables with $\mathrm{p}<0.2$ in the univariate analyses. OR, odds ratio; CI, confidence interval; RRRS, Risk-Rescue Rating Scale; SIS, Suicide Intent Scale.

than that of the SIS ( 0.715 vs. $0.653, p=0.085)$ (Fig. 1C).

The optimal cutoff values for the individual psychological scales for predicting hospitalization were 37.5 for the RRRS, 12.0 for the SIS, and 4.0 for the SIS part 2. At these respective cutoff values, the sensitivity of the RRRS and SIS part 2 for predicting hospitalization was higher than the specificity; the sensitivity and specificity were, respectively, $75.7 \%$ and 

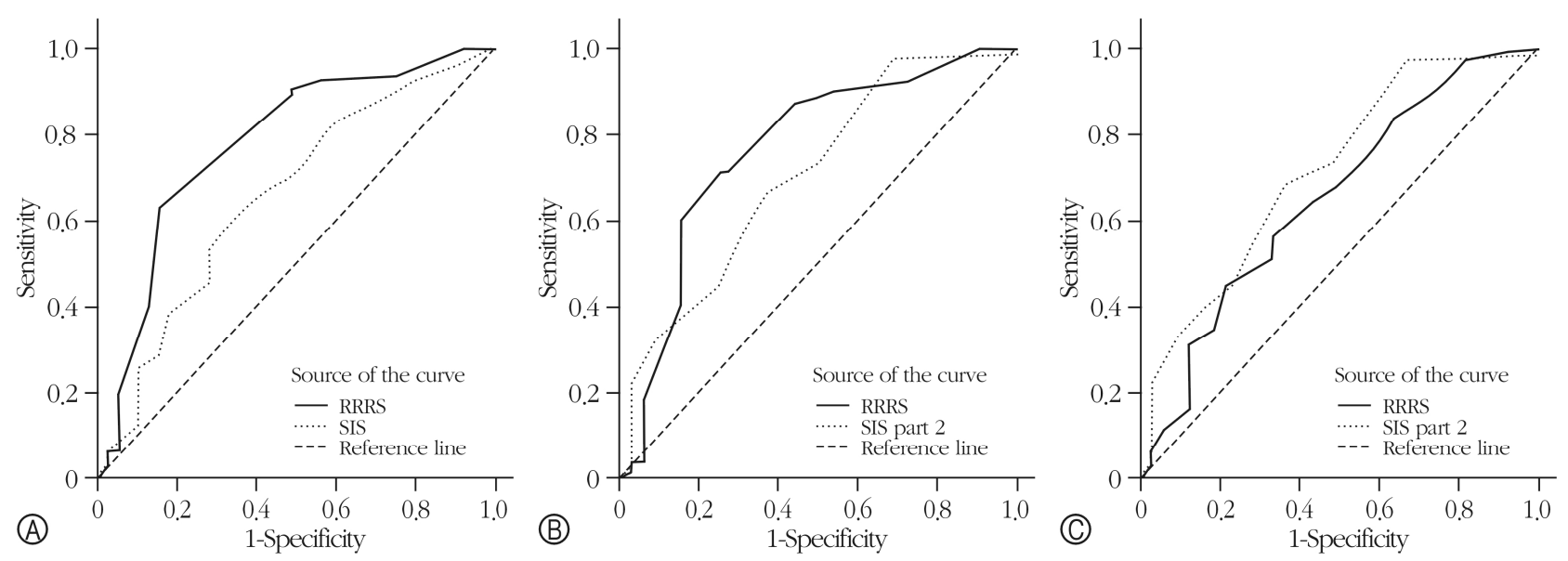

Fig. 1. The discriminative performance in predicting hospitalization between psychological scales. The RRRS, SIS, and SIS part 2 were administered to 166, 158, and 129 patients, respectively. (A) The predictive performance of the RRRS was significantly higher than that of the SIS $(0.777$ [95\% CI, 0.686-0.869] vs. 0.662 [95\% CI, 0.556-0.763], $\mathrm{p}=0.042)$. The predictive performance of the SIS part 2 was comparable to that of the RRRS $(0.703$ [95\% CI, 0.592-0.813] vs. 0.765 [95\% CI, 0.659-0.864], p=0.315) (B), and tended to be higher than that of the SIS (0.715 [95\% CI, 0.608-0.821] vs. 0.653 [95\% CI, 0.539-0.756], p=0.085) (C). CI, confidence interval; RRRS, Risk-Rescue Rating Scale; SIS, Suicide Intent Scale.

$68.2 \%$ for the RRRS, $60.5 \%$ and $64 \%$ for the SIS, and $72.8 \%$ and $52.5 \%$ for the SIS part 2.

\section{DISCUSSION}

Approaching a suicide attempter is an exceedingly difficult and complicated task, with inherent unpredictability and uncertainty. It is even more challenging for clinicians to manage elderly suicide attempters, because old age itself may complicate SA risk assessment and increase vulnerability to injury caused by suicidal behavior. The final stage of acute care for a suicide attempter in the ED is the clinician's decision of whether to hospitalize, a decision made based on the SA's severity, assessed by degree of suicidal lethality and intent ${ }^{11,16)}$. These 2 factors, known to be age-related, are important determinants of hospitalization ${ }^{5,11,12)}$. However, few studies have evaluated their utility as predictors of hospitalization in elderly suicide attempters; most studies focus primarily on using the dimensions of suicidal lethality and intent to assess the risk of suicide, repetitive SAs, and mortality related to $S A s^{18,19,21,22)}$. To our knowledge, this is the first study to simultaneously compare the predictive ability of the psychological scales reflecting suicide lethality (RRRS) and intent (SIS) for hospitalization in elderly suicide attempters.

The first main finding of this study is that, as expected, both the RRRS and SIS, with significantly higher scores in the hospitalization group, can be considered independent predictors of hospitalization in elderly suicide attempters.

The second main finding is that administration of the RRRS is a more useful predictor of hospitalization than the SIS in elderly suicide attempters. Regarding predictive perform- ance, the RRRS showed a significantly higher AUC than did the SIS. This result seems to be associated with the specific setting of the ED, in which this study was conducted. The need for appropriate care in the ED is a function of disorder perception or the immediacy with which patients require acute therapy and stabilization ${ }^{25)}$. Moreover, it has been reported that ED clinicians may focus more generally on treating medical/physical crises rather than the psychological distress of suicide attempters ${ }^{26}$. From these perspectives, clinicians prioritize stabilization of acute injuries following SAs, and as a result, suicide lethality might be judged a more dominant determinant of hospitalization in an emergency setting. This perspective seems more prominent in the elderly population owing to their high vulnerability to medical/physical injuries. This was illustrated by the fact that $82 \%$ of hospitalized elderly patients in this study were preferentially admitted to the medical/surgical ward for extension of therapeutic interventions performed in the ED before referral for psychiatric care. The RRRS additionally exhibited higher sensitivity than specificity for predicting the need for hospitalization at its optimal cutoff value, whereas the SIS did not. Considering the priority of sensitivity over specificity owing to the focus of this study on prediction of patients requiring hospitalization, this finding further supports the clinical utility of the RRRS as a predictor of hospitalization.

The SIS, which incorporates objective circumstances (SIS part 1) and self-report questionnaires (SIS part 2), is known to have limitations regarding poor agreement between its subscales ${ }^{12,20)}$. This point has been well observed in previous factorial analysis studies of other age populations. Diaz et al. ${ }^{21)}$ reported that 6 of 7 items of the SIS part 2 and only 
1 of 8 items of the SIS part $1(1 / 8)$ yielded positive factor loading for the expected lethality; furthermore, Baca-García et al. ${ }^{27)}$ found that an independent effect on the increased likelihood of hospitalization was demonstrated for only 4 items of the SIS part 2 and not for any items of the SIS part 1. Because we did not conduct factor analyses of the psychological scales, the descriptions of these properties for this study population are necessarily limited. However, although these age-oriented findings are limited in their application to elderly individuals, we consider that this incongruity between subscales partially contributes to the low predictive performance of the SIS for hospitalization in the ED.

Another remarkable finding of this study concerns the predictive ability of the SIS subscales for hospitalization in elderly suicide attempters. Based on the incongruity between the SIS subscales, we assumed that their predictive ability for hospitalization might differ. This study identified only the SIS part 2 as an independent predictor of hospitalization, with higher sensitivity than specificity. Furthermore, for predicting hospitalization, its performance tended to be superior to that of the SIS and comparable to that of the RRRS. These results are in close agreement with the findings of previous studies ${ }^{21,27)}$. Additionally, the RRRS was more strongly correlated with the SIS part 2 than the SIS in this study (RRRS and SIS part 2: $r=0.424, p=0001$; RRRS and SIS: $r=0.255$, $p<0.001)$, a finding that emphasizes the relatively high prediction potential of the SIS part 2 for lethality revealed by Diaz et al. ${ }^{21)}$ Given that lethality is the more influential dimension in emergency settings, and that a high correlation between lethality and intent is found in elderly suicide attempters, we infer that the SIS part 2 might be a valuable tool for predicting hospitalization of elderly suicide attempters in the ED. However, although these findings support the validity of the SIS part 2 in predicting hospitalization, the validity of its exclusive use as a predictive tool must be confirmed through further investigation; its feasibility is limited in the ED because it consists of self-report questionnaires prone to influence from the patient's conditions.

Before performing this study, we anticipated that the degrees of suicide lethality and intent might be high because the criteria for recruitment is subject to old age, and increasing age is associated with increasing degrees of these factors, as mentioned earlier. However, the degree of increment was more pronounced in the RRRS than the SIS; the mean RRRS score of hospitalized patients was much higher, whereas the mean SIS score was similar to those of previous studies of other age populations ${ }^{21,28-30}$. The reason that both scales showed this discrepancy in increment value seems to be associated with the characteristics of the subjects included in the study. In the present study, compared with others, the proportions of patients using a fatal SA method, using alcohol during their $\mathrm{SA}$, and experiencing depression were relatively high. Alcohol use and depression are known to increase suicidal lethality related to the use of fatal methods (indicated by higher RRRS scores) ${ }^{30-32)}$. Moreover, alcohol use is known to aggravate the impulsivity of suicidal behaviors that are not preplanned or intentional (lower SIS scores) ${ }^{33,34)}$. The relationship between depression and impulsivity in suicidal behavior remains unclear; mixed results have been reported, and some growing evidence supports a direct correlation between the two ${ }^{35-37}$. Although the effect of these clinical characteristics on suicide lethality and intent could not be ascertained beyond any doubt, we considered that they might have contributed to a higher increase in the RRRS scores than the SIS scores in a considerable proportion. Overall, our findings based on these relationships seem to provide further support for the RRRS being a more powerful scale than the SIS in predicting hospitalization of elderly suicide attempters, considering that a high prevalence of depression, alcohol dependence, and use of lethal methods was observed in the elderly population ${ }^{38}$. However, the discriminative incremental degree of both scales could be explained by the effect of other clinical variables, and further surveys are required to explore what these could be.

There are several limitations to be considered in this study. First and primarily, our study employed a retrospective design, which has inherent problems, including the potential for incomplete data and recall bias. To minimize these weaknesses, thorough data collection was conducted through structured questionnaire interviews by trained researchers as well as review of medical records and use of multiple informants. Additionally, the number of patients administered the SIS part 2 was relatively small, which risks selection bias. Therefore, we tried to include as many of those patients as possible within the last 5 years, and the analytic focus with respect to this scale was designed to compare with other scales. Second, despite the importance of suicide lethality as a more dominant factor affecting hospitalization in emergency settings, we did not consider its effect on differences in assigned facilities for hospitalization. Further investigation of each patient admitted to each separate facility type (i.e., the medical/surgical and psychiatric wards) is needed. Third, the characteristics of SAs reportedly differ according to the degree of aging in the elderly population, such as the young-old, old-old, and oldest-old ${ }^{39,40}$. However, such elderly populations in the present study were considered as one group. Furthermore, to apply our results to clinical practice and evaluate the usefulness of the scales for elderly patients, we considered that the psychological scales' predictive performance for hospitalization could be confirmed through comparative investigation with other age groups, including young and adult populations. Finally, because this study was conducted at the ED of a tertiary-care hospital, our findings may not be generalizable to other ED settings with different systems 
for managing elderly suicide attempters.

In conclusion, suicide lethality and intent are crucial dimensions affecting the decision to hospitalize, and are representative factors explaining the characteristics of elderly suicide attempters. In the present study, we examined the evaluation and disposition of elderly suicide attempters in the ED, focusing on the psychological scales reflecting suicide lethality and intent. Our results showed that the RRRS and SIS scores were independent predictors of hospitalization in elderly suicide attempters. However, given that ED settings prioritize acute stabilization of injuries, the RRRS was a more useful and powerful scale for predicting hospitalization in elderly suicide attempters with respect to performance and diagnostic accuracy. Additionally, although the predictive utility of the SIS part 2 for hospitalization was also independently substantial, its application in clinical settings appears limited based on its feasibility.

Many factors affect clinical judgment of whether to hospitalize. ED clinicians may highlight the patient's symptoms when making their decision, rather than considering the patient's demographic and psychosocial factors ${ }^{28,30)}$. In this sense, although the merit of psychological scales can never surpass the clinician's judgment regarding hospitalization, because these scales were devised to measure a patient's symptoms in a standardized manner, they may be used as complements to the clinician's judgment in emergency settings ${ }^{22}$. In particular, the utility of the scales is considered more prominent in elderly suicide attempters, who are medically/physically and mentally vulnerable after their suicide behaviors.

Conflicts of Interest Disclosures: The researchers claim no conflicts of interest.

\section{Acknowledgments}

The authors are appreciative of the helpful assistance of Ji Hyeon Huh and Na Ri Kim (interview researchers) in collecting data using face-to-face interviews and questionnaires.

\section{REFERENCES}

1. Värnik P. Suicide in the world. Int J Environ Res Public Health 2012;9:760-71.

2. World Health Organization (WHO). First WHO report on suicide prevention [Internet]. Geneva: WHO; c2014 [cited 2017 Jun 1]. Available from: http://www.who.int/mediacentre/news/ releases/2014/suicide-prevention-report/en/.

3. Kim KH, Jeong KY, Lee JS, Choi HS, Hong HP, Ko YG. The characteristics of elderly patients with suicide attempts: a comparative study with non-elderly patients. Ann Geriatr Med Res 2016;20:209-20.

4. Van Orden K, Conwell Y. Suicides in late life. Curr Psychiatry Rep 2011;13:234-41.
5. Miret M, Nuevo R, Morant C, Sainz-Cortón E, Jiménez-Arriero MA, López-Ibor JJ, et al. Differences between younger and older adults in the structure of suicidal intent and its correlates. Am J Geriatr Psychiatry 2010;18:839-47.

6. World Health Organization (WHO). Chapter 1: Global Health: today's challenges [Internet]. Geneva: WHO; c2003 [cited 2017 Jun 1]. Available from: http://www.who.int/whr/2003/chapter1/ en/index 3.html.

7. Drew BL, Jones SL, Meldon SW, Varley JD. Emergency department visits for suicidality in three hospitals. Arch Psychiatr Nurs 2006;20:117-25.

8. Waterhouse J, Platt S. General hospital admission in the management of parasuicide. A randomised controlled trial. Br J Psychiatry 1990;156:236-42.

9. Reid WH. Prognosis after suicide attempt: standard of care and the consequences of not meeting it. J Psychiatr Pract 2009;15: 141-4.

10. Troister T, Links PS, Cutcliffe J. Review of predictors of suicide within 1 year of discharge from a psychiatric hospital. Curr Psychiatry Rep 2008;10:60-5.

11. Practice guideline for the assessment and treatment of patients with suicidal behaviors. Am J Psychiatry 2003;160(11 Suppl): 1-60.

12. Hamdi E, Amin Y, Mattar T. Clinical correlates of intent in attempted suicide. Acta Psychiatr Scand 1991;83:406-11.

13. Liotta M, Mento C, Settineri S. Seriousness and lethality of attempted suicide: a systematic review. Aggress Violent Behav 2015;21:97-109.

14. Weisman AD, Worden JW. Risk-rescue rating in suicide assessment. Arch Gen Psychiatry 1972;26:553-60.

15. Beck AT, Schuyler D, Herman I. Development of suicidal intent scales. In: Beck AT, Resnik HL, Lettieri DJ, editors. The prediction of suicide. Bowie (MD): Charles Press; 1974. p. 45-56.

16. Haw C, Hawton K, Houston K, Townsend E. Correlates of relative lethality and suicidal intent among deliberate self-harm patients. Suicide Life Threat Behav 2003;33:353-64.

17. Goldberg JF, Ernst CL, Bird S. Predicting hospitalization versus discharge of suicidal patients presenting to a psychiatric emergency service. Psychiatr Serv 2007;58:561-5.

18. Harriss L, Hawton K. Suicidal intent in deliberate self-harm and the risk of suicide: the predictive power of the Suicide Intent Scale. J Affect Disord 2005;86:225-33.

19. Stefansson J, Nordström P, Jokinen J. Suicide Intent Scale in the prediction of suicide. J Affect Disord 2012;136:167-71.

20. Antretter E, Dunkel D, Haring C, Corcoran P, De Leo D, Fekete $S$, et al. The factorial structure of the Suicide Intent Scale: a comparative study in clinical samples from 11 European regions. Int J Methods Psychiatr Res 2008;17:63-79.

21. Diaz FJ, Baca-Garcia E, Diaz-Sastre C, García Resa E, Blasco $\mathrm{H}$, Braquehais Conesa D, et al. Dimensions of suicidal behavior according to patient reports. Eur Arch Psychiatry Clin Neurosci 2003;253:197-202.

22. Misson H, Mathieu F, Jollant F, Yon L, Guillaume S, Parmentier C, et al. Factor analyses of the Suicidal Intent Scale (SIS) and the Risk-Rescue Rating Scale (RRRS): toward the identification of homogeneous subgroups of suicidal behaviors. J Affect Disord 2010;121:80-7.

23. O'Carroll PW, Berman AL, Maris RW, Moscicki EK, Tanney 
BL, Silverman MM. Beyond the Tower of Babel: a nomenclature for suicidology. Suicide Life Threat Behav 1996;26:237-52.

24. Pedinielli JL, Delahousse J, Chabaud B. The "lethality" of attempted suicide. Ann Med Psychol (Paris) 1989;147:535-50.

25. Gruneir A, Silver MJ, Rochon PA. Emergency department use by older adults: a literature review on trends, appropriateness, and consequences of unmet health care needs. Med Care Res Rev 2011;68:131-55.

26. Pompili M, Girardi P, Ruberto A, Kotzalidis GD, Tatarelli R. Emergency staff reactions to suicidal and self-harming patients. Eur J Emerg Med 2005;12:169-78.

27. Baca-García E, Diaz-Sastre C, Resa EG, Blasco H, Conesa DB, Saiz-Ruiz J, et al. Variables associated with hospitalization decisions by emergency psychiatrists after a patient's suicide attempt. Psychiatr Serv 2004;55:792-7.

28. Kumar CT, Mohan R, Ranjith G, Chandrasekaran R. Characteristics of high intent suicide attempters admitted to a general hospital. J Affect Disord 2006;91:77-81.

29. Grøholt B, Ekeberg O, Haldorsen T. Adolescents hospitalised with deliberate self-harm: the significance of an intention to die. Eur Child Adolesc Psychiatry 2000;9:244-54.

30. Harriss L, Hawton K, Zahl D. Value of measuring suicidal intent in the assessment of people attending hospital following selfpoisoning or self-injury. Br J Psychiatry 2005;186:60-6.

31. Sher L. Alcohol consumption and suicide. QJM 2006;99:57-61.

32. Grunebaum MF, Galfalvy HC, Oquendo MA, Burke AK, Mann JJ. Melancholia and the probability and lethality of suicide attempts. Br J Psychiatry 2004;184:534-5.
33. Lejoyeux M, Huet F, Claudon M, Fichelle A, Casalino E, Lequen V. Characteristics of suicide attempts preceded by alcohol consumption. Arch Suicide Res 2008;12:30-8.

34. Suokas J, Lönnqvist J. Suicide attempts in which alcohol is involved: a special group in general hospital emergency rooms. Acta Psychiatr Scand 1995;91:36-40.

35. Kim J, Lee KS, Kim DJ, Hong SC, Choi KH, Oh Y, et al. Characteristic risk factors associated with planned versus impulsive suicide attempters. Clin Psychopharmacol Neurosci 2015;13: 308-15.

36. Dumais A, Lesage AD, Alda M, Rouleau G, Dumont M, Chawky $\mathrm{N}$, et al. Risk factors for suicide completion in major depression: a case-control study of impulsive and aggressive behaviors in men. Am J Psychiatry 2005;162:2116-24.

37. Corruble E, Damy C, Guelfi JD. Impulsivity: a relevant dimension in depression regarding suicide attempts? J Affect Disord 1999;53:211-5.

38. Lebret S, Perret-Vaille E, Mulliez A, Gerbaud L, Jalenques I. Elderly suicide attempters: characteristics and outcome. Int J Geriatr Psychiatry 2006;21:1052-9.

39. Paraschakis A, Douzenis A, Michopoulos I, Christodoulou C, Vassilopoulou K, Koutsaftis F, et al. Late onset suicide: distinction between "young-old" vs. "old-old" suicide victims. How different populations are they? Arch Gerontol Geriatr 2012;54: 136-9.

40. O'Connell H, Chin AV, Cunningham C, Lawlor BA. Recent developments: suicide in older people. BMJ 2004;329:895-9. 